\title{
Distributed Energy Management of the Hybrid AC/DC Microgrid with High Penetration of Distributed Energy Resources Based on ADMM
}

\author{
Yaling Chen $\mathbb{D}^{1},{ }^{1}$ Luxi Hao $\mathbb{D}^{2},{ }^{2}$ and Gaowen Yin $\mathbb{1}^{3}$ \\ ${ }^{1}$ Strategic Emerging Industries Development Research Centers, Business School of Hunan University, Changsha 410208, China \\ ${ }^{2}$ State Grid Hunan Electric Power Company Limited Research Institute, Changsha 410000, China \\ ${ }^{3}$ School of Electrical and Information Engineering, Hunan University, Changsha 410082, China
}

Correspondence should be addressed to Yaling Chen; dorianchen@hnu.edu.cn

Received 25 August 2021; Accepted 3 September 2021; Published 15 September 2021

Academic Editor: Chun Wei

Copyright (C) 2021 Yaling Chen et al. This is an open access article distributed under the Creative Commons Attribution License, which permits unrestricted use, distribution, and reproduction in any medium, provided the original work is properly cited.

This paper aims to investigate energy management of the hybrid AC/DC microgrid with the high penetration of distributed energy resources (DERs), such as electrical vehicles, heat pumps, and photovoltaics. In the previous studies, energy management of the hybrid microgrid is usually carried out by the system operator in a centralized manner, which suffers from the compromise of privacy information protection and the risk of single-point failure. Therefore, this paper proposes a distributed energy management scheme of the hybrid microgrid using the projection function-based alternating direction method of multipliers (PADMM), which allows each subgrid, i.e., AC subgrid and DC subgrid, to make day-ahead schedules independently with information exchanges while obtaining the optimal energy management solution. The energy management problem of the hybrid microgrid is formulated as a mixed-integer quadratic programming (MIQP) model, considering DER and energy storage system operation constraints, system operation constraints, and converter operation constraints. Then, the MIQP model is decomposed and distributed into smaller-scale QP models between subgrids using the P-ADMM algorithm, which can handle binary variables through projection functions. The numerical results conducted on the hybrid microgrid demonstrate that the proposed distributed scheme can effectively achieve optimal energy management for the hybrid AC/DC microgrid in a distributed manner.

\section{Introduction}

The next-generation distribution system involves the massive deployment of distributed energy resources (DERs), such as electrical vehicles (EVs), heat pumps (HPs), and photovoltaics (PVs) [1]. Adopting the microgrid concept (a small-scale autonomous energy system) has become an effective and promising technology for the integration of DERs due to its technical and economic benefits including the lower operation cost, higher reliability, and stronger resiliency [2]

Generally, microgrids can be put into three main categories according to the voltage type: (1) AC microgrids, (2) DC microgrids, and (3) hybrid AC/DC microgrids [3]. The hybrid AC/DC microgrid separates the AC and DC power supplies and loads, with the AC bus and DC bus linked through a bidirectional converter (BC) [4]. Compared with the conventional $\mathrm{AC}$ microgrid requiring a number of $\mathrm{AC} /$ DC converters to convert power, the hybrid AC/DC microgrid has the lower equipment investment and can reduce the energy loss during power conversion. Therefore, the hybrid AC/DC microgrid has been considered as an important type of microgrids as more DC power sources and loads are connected [5].

Although the utilization of the microgrid brings about technical and economic benefits, there exist challenges on the microgrid operation, such as energy management. Energy management refers to the optimized operation of dispatchable resources within microgrids such as controllable generation units and loads in order to realize the best tech-economic operation of the microgrid based on system information and forecast data [6]. 
Energy management for AC or DC microgrids has been widely studied. In [6], a double-layer coordinated energy management approach was proposed for microgrids considering grid-connected and islanding modes, which consists of two layers: the schedule layer obtains an economic operation scheme based on forecast data, while the dispatch layer dispatches controllable units based on real-time data. A centralized optimal microgrid scheduling model which considers multiperiod islanding constraints was proposed in [7]. The optimal scheduling model is solved using the bender decomposition technique, and the obtained solution ensures the microgrid has sufficient capacity to switch to the islanded mode if needed by examining the solution with a proposed islanding criterion. In [8], a practical energy management model for microgrids which considers the operational constraints of DERs, active-reactive power balance, unbalanced system configuration, and voltage-dependent loads was formulated. Moreover, a novel linearization approach was used to reduce the computation complexity. In [9], a model predictive control-based microgrid energy management framework was proposed, which considers the demand response in smart loads and models power flow and unit commitment constraints simultaneously. A multiobjective optimal scheduling model for a DC microgrid considering PVs and EVs was formulated in [10] and solved by the NSGA-II algorithm. In [11], energy management of a DC microgrid with energy storage systems (ESSs) and EVs was studied. Moreover, the uncertainty of renewable energy resources was widely studied and modeled in the microgrid energy management problem in [12-14].

For hybrid AC/DC microgrids, the energy management problem of the hybrid microgrid was modeled in [14] considering multiple distributed ESSs. In [15], a decentralized power sharing method was proposed to share power demands between the AC and DC sources without requiring communication between power sources. A temporally coordinated energy management strategy for the hybrid microgrid was proposed in [16] to deal with uncertainties of the RESs, loads, and converters while considering the dynamic conversion efficiency model of the converter. A twostage min-max-min robust optimal energy management for the hybrid microgrid was proposed in [17] to deal with the uncertainties of generation sources and loads. An up-down energy management scheme of the hybrid microgrid was proposed in [18], which consists of two levels: the generation and load balance are realized at the system level, while power converter-based resources are used to control the voltage variations at the device level. However, energy management of the hybrid microgrid in the aforementioned studies is carried out by the system operator in a centralized manner, which suffers from the compromise of privacy information protection and single-point failure risk.

To resolve the aforementioned issues, distributed methods, such as analytical target cascading (ATC) and alternating direction method of multipliers (ADMM), have been widely applied to solve optimization models in a distributed/decentralized manner in order to design distributed/decentralized schemes. For instance, in [19-22], the ATC method was used. In [19], a decentralized data-driven load restoration scheme was proposed for the coupled transmission and distribution system based on ATC. The uncertainty of wind power and load consumption was dealt with by formulating a data-driven robust model. In [20], an ATC-based decentralized generator startup sequence scheme was proposed for bulk system restoration considering the available black-start resources in the distribution system. In [21], a spatiotemporal decomposition and coordination scheme was proposed for load restoration in an AC/DC hybrid based on the ATC algorithm. In [22], the ADMM algorithm was used to solve the local flexibility market clearing problem and to design a distributed market clearing scheme.

For the energy management of the microgrid, in [23], a distributed energy management scheme was proposed for the AC microgrid based on the predictor-corrector proximal multiplier method. In the proposed scheme, the local controller of each unit (DERs and loads) optimizes its cost function and sends an optimal schedule to the centralized controller, and the centralized controller optimizes the total cost function based on the system operational constraints. However, the hybrid AC/DC configuration is not considered. In [24], a distributed robust energy management scheme for networked hybrid AC/DC microgrids was proposed, in which each hybrid AC/DC microgrid operator solves the adjustable robust optimization model to optimize its operational cost considering the uncertainty of the renewable generation and load demand. For the networked microgrids, the coordination of energy sharing is carried out to minimize the power loss based on the alternating direction method of multipliers (ADMM). However, in order to use the ADMM to solve the energy management model in a distributed manner, the model was reformulated as a convex model by removing nonlinear terms based on strict assumptions regarding the structure of the objective function, which makes the proposed distributed energy management only suitable for specific models. In order to resolve the aforementioned issues, we propose a distributed energy management scheme for the hybrid AC/DC microgrid based on the projection function-based alternating direction method of multipliers (P-ADMM) that can deal with binary variables efficiently. The P-ADMM algorithm has been widely used to solve nonconvex instances. In [25-28], the P-ADMM algorithm was used to solve the service restoration problem of distribution networks in a hierarchical/ distributed manner. In [29], a P-ADMM-based distributed scheme was proposed to coordinate the fast dispatch of PV inverters with the slow dispatch of on-load tap changer and capacitor banks for voltage regulation in unbalanced distribution systems. The practical evidence in these studies denotes that the P-ADMM is an effective method to obtain quality solutions for mixed-integer quadratic programming (MIQP) and mixed-integer linear programming (MILP) models.

In this study, the energy management problem of the hybrid microgrid is firstly formulated as a MIQP model, considering DER and ESS operation constraints, system operation constraints, and converter operation constraints. Then, the MIQP model is decomposed and distributed into 
smaller-scale submodels using the P-ADMM-based algorithm that can handle binary variables through projection functions. In the proposed scheme, each subgrid, i.e., AC subgrid and DC subgrid, can make day-ahead schedules independently with information exchanges while achieving optimal energy management.

The main contributions of this paper are summarized as follows: (1) proposes a distributed energy management scheme for the hybrid AC/DC microgrid, which allows each subgrid to make energy schedules independently with information exchanges while realizing optimal energy management and (2) proposes a P-ADMM-based algorithm to efficiently solve the MIQP energy management model in a distributed manner.

The rest of this paper is organized as follows. The framework of the proposed distributed energy management scheme and energy management model formulations are presented in Section 2. The P-ADMM-based algorithm to solve the energy management model is presented in Section 3. Case studies are presented and discussed in Section 4, followed by conclusions.

\section{Framework and Model Formulations of the Distributed Energy Management Scheme}

\subsection{Framework of the Distributed Energy Management} Scheme. Figure 1 illustrates the physical and communication architecture of the hybrid AC/DC microgrid, to which various sources and loads are connected. In the DC subgrid, photovoltaics (PVs), ESSs, EVs, and heat pumps (HPs) are connected, while diesel generators (DGs) and conventional AC loads are connected to the AC subgrid. AC subgrid and DC subgrid are tied by a BC.

In the proposed distributed scheme, the AC subgrid operator and DC subgrid operator communicate with each other to make day-ahead energy schedules for respective sources and loads independently.

2.2. Optimal Energy Management Model. In this section, the optimal energy management model of the hybrid microgrid is formulated.

2.2.1. Objective Function. For the energy management of microgrids, the objective function is to minimize operational costs during the scheduling period. The objective function is formulated as follows:

$$
\min \sum_{t \in N_{T}} c_{1}^{G}\left(p_{t}^{\mathrm{DG}}\right)^{2}+c_{2}^{G} p_{t}^{\mathrm{DG}}+\lambda_{t} p_{t}^{\mathrm{UG}}+b\left(p_{t}^{\mathrm{UG}}\right)^{2} .
$$

The objective function costs of three terms are as follows: the first two terms are to minimize energy costs of DGs, where $N_{T}$ is the set of scheduling periods, $c_{1}^{\mathrm{G}}$ and $c_{2}^{\mathrm{G}}$ are generation cost parameters, and $p_{t}^{\mathrm{DG}}$ is the DG generation; the last term is to minimize the cost of purchasing energy from the external grid, where $\lambda_{t}$ is the energy sport price, $b$ is the price sensitivity coefficient, and $p_{t}^{\mathrm{UG}}$ is the purchased power.

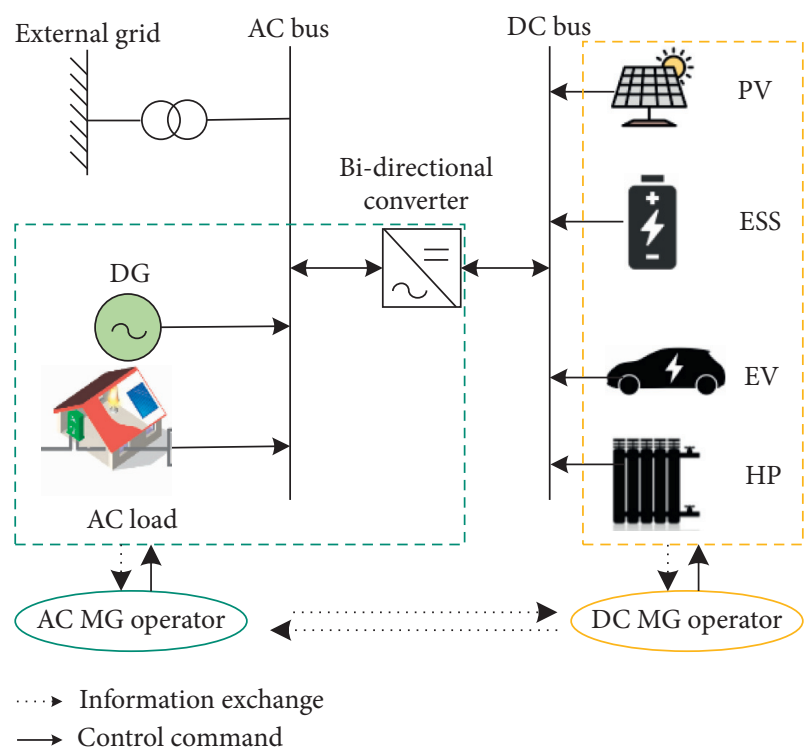

FIgURe 1: Physical and communication architecture of the hybrid AC/DC microgrid.

\subsubsection{Power Balance Equations}

$$
\begin{gathered}
p_{t}^{\mathrm{UG}}+p_{t}^{\mathrm{DG}}+\eta_{\mathrm{dta}} p_{t}^{\mathrm{dta}}=p_{t}^{\mathrm{AC}-L}+p_{t}^{\mathrm{atd}}, \quad \forall t \in N_{T}, \\
p_{t}^{\mathrm{ed}}+p_{t}^{\mathrm{pv}}+\eta_{\mathrm{atd}} p_{t}^{\mathrm{atd}}=p_{t}^{\mathrm{ec}}+\sum_{i \in N_{e}} p_{i, t}^{\mathrm{ev}}+\sum_{i \in N_{h}} p_{i, t}^{\mathrm{hp}}+p_{t}^{\mathrm{dta}}, \quad \forall t \in N_{T} .
\end{gathered}
$$

Equations (2) and (3) are active power balance equations of the AC subgrid and DC subgrid, respectively, where $N_{h}$ and $N_{e}$ are sets of HPs and EVs, respectively; $p_{t}^{\mathrm{dta}}$ is the power transferring from the DC side to the AC side at hour $t$ and $p_{t}^{\text {atd }}$ is the power transferring from the AC side to the DC side at hour $t ; p_{t}^{\mathrm{AC}-\mathrm{L}}$ is the conventional AC load consumption; $p_{i, t}^{\text {ev }}$ is the EV charging power of the $i$ th $\mathrm{EV}$ at hour $t ; p_{i, t}^{\mathrm{hp}}$ is the power consumption of the $i$ th heat pump at hour $t$; $p_{t}^{\mathrm{ec}}$ and $p_{t}^{\mathrm{ed}}$ are the ESS charging and discharging power, respectively; $p_{t}^{\mathrm{pv}}$ is the PV generation power; and $\eta_{\mathrm{dta}}$ and $\eta_{\text {atd }}$ are power transfer coefficients.

\subsubsection{DG Operation Constraints}

$$
\begin{aligned}
& p_{t}^{\mathrm{DG}, \min } \leq p_{t}^{\mathrm{DG}} \leq p_{t}^{\mathrm{DG}, \max }, \quad \forall t \in N_{T}, \\
& \left\{\begin{array}{l}
p_{t}^{\mathrm{DG}, \mathrm{dr}} \leq p_{t}^{\mathrm{DG}}-p_{t-1}^{\mathrm{DG}} \leq p_{t}^{\mathrm{DG}, \text { ur }} \\
p_{t_{1}}^{\mathrm{DG}, \mathrm{dr}} \leq p_{t_{1}}^{\mathrm{DG}}-p_{0}^{\mathrm{DG}} \leq p_{t_{1}}^{\mathrm{DG}, \mathrm{ur}}, \quad \forall t \in N_{T} .
\end{array}\right.
\end{aligned}
$$

Constraint (4) is the DG capacity constraint, where $p_{t}^{\mathrm{DG}, \max }$ and $p_{t}^{\mathrm{DG}, \min }$ are the maximum and minimum limits of the DG active power generation. Constraint (5) is the DG ramping constraint, where $p_{t}^{\mathrm{DG} \text {,ur }}$ and $p_{t}^{\mathrm{DG}, \mathrm{dr}}$ are the upramping and downramping limits. 


\subsubsection{EV Operation Constraints}

$$
e_{i, t}^{\min } \leq e_{i, 0}+\sum_{t_{-} \leq t}\left(p_{i, t_{-}}^{\mathrm{ev}}-d_{i, t_{-}}^{\mathrm{ev}}\right) \leq e_{i, t}^{\max }, \quad \forall i \in N_{e}, t \in N_{t},
$$

$$
p_{i, t}^{\mathrm{ev}, \min } \leq p_{i, t}^{\mathrm{ev}} \leq a_{i, t} p_{i, t}^{\mathrm{ev}, \max }, \quad \forall i \in N_{e}, t \in N_{t}
$$

Constraint (6) is the EV SOC level constraint, where $d_{i, t}^{\mathrm{ev}}$ is the power consumption of the ith $\mathrm{EV}$ at hour $t$ due to driving, $e_{i, 0}$ is the initial SOC level of the $i$ th $\mathrm{EV}$, and $e_{i, t}^{\min }$ and $e_{i, t}^{\max }$ are the minimum and maximum SOC level limits of the $i$ th EV. Constraint (7) is the EV charging power constraint, where $p_{i, t}^{\text {ev,min }}$ and $p_{i, t}^{\text {ev,max }}$ are the minimum and maximum charging power limits of the $i$ th EV at hour $t$ and $a_{i, t}$ is the charging availability of the ith EV at hour $t$.

\subsubsection{HP Operation Constraints}

$$
\begin{aligned}
& c_{i}^{\mathrm{cop}} p_{i, t}^{\mathrm{hp}}-k_{1}\left(K_{i, t}^{h}-K_{i, t}^{u}\right)-k_{2}\left(K_{i, t}^{h}-K_{i, t}^{s}\right)=k_{3}\left(K_{i, t}^{h}-K_{i, t-1}^{h}\right), \quad \forall t \in N_{T}, i \in N_{h}, \\
& -k_{4}\left(K_{i, t}^{s}-K_{i, t}^{u}\right)+k_{2}\left(K_{i, t}^{h}-K_{i, t}^{s}\right)=k_{5}\left(K_{i, t}^{s}-K_{i, t-1}^{s}\right), \quad \forall t \in N_{T}, i \in N_{h}, \\
& K_{i, t}^{h, \min } \leq K_{t, i}^{h} \leq K_{i, t}^{h, \max }, \quad \forall t \in N_{T}, i \in N_{h}, \\
& p_{i, t}^{\mathrm{hp}, \min } \leq p_{i, t}^{\mathrm{hp}} \leq p_{i, t}^{\mathrm{hp}, \max }, \quad \forall t \in N_{T}, i \in N_{h} .
\end{aligned}
$$

Constraints (8) and (9) represent thermal equations of the house equipped with the HP $[30,31]$, where $c_{i}^{\text {cop }}$ is the coefficient of performance; $k_{1}, k_{2}, k_{3}, k_{4}$, and $k_{5}$ are thermal efficiency coefficients; and $K_{i, t}^{h}, K_{i, t}^{u}$, and $K_{i, t}^{s}$ are the household inside temperature, outside temperature, and structure temperature, respectively. Constraint (10) represents the household temperature constraint, where $K_{i, t}^{h \text {, } m a x}$ and $K_{i, t}^{h, \text { min }}$ are the maximum and minimum limits of the household inside temperature. Constraint (11) represents the HP power consumption constraint, where $p_{i, t}^{h p, \text { max }}$ and $p_{i, t}^{h p \text {, min }}$ are the maximum and minimum limits of the $i$ th heat pump power consumption at hour $t$.

\subsubsection{ESS Operation Constraints}

$$
\begin{aligned}
& x_{t}^{\mathrm{ec}} p_{i}^{\mathrm{ec}, \mathrm{min}} \leq p_{t}^{\mathrm{ec}} \leq x_{t}^{\mathrm{ec}} p_{i}^{\mathrm{ec}, \max }, \quad \forall t \in N_{T}, \\
& x_{t}^{\mathrm{ed}} p_{i}^{\mathrm{ed}, \mathrm{min}} \leq p_{t}^{\mathrm{ed}} \leq x_{t}^{\mathrm{ed}} p_{i}^{\mathrm{ed}, \max }, \quad \forall i \in N_{e}, t \in N_{T}, \\
& x_{i, t}^{\mathrm{ec}}+x_{i, t}^{\mathrm{ed}}=1, \quad t \in N_{T}, \\
& E_{t}^{\mathrm{es}}=E_{t-1}^{\mathrm{es}}+\eta^{c} p_{t}^{\mathrm{ec}} \Delta t-\frac{1}{\eta^{d}} p_{t}^{\mathrm{ed}} \Delta t, \quad \forall t \in N_{T}, \\
& E_{t}^{\mathrm{es}, \min } \leq E_{t}^{\mathrm{es}} \leq E_{t}^{\mathrm{es}, \max } .
\end{aligned}
$$

Constraints (12) and (13) are ESS charging and discharging power constraints, respectively, where $p_{i}^{\mathrm{ec}, \min }$ and $p_{i}^{\mathrm{ec}, \max }$ are the minimum and maximum limits of ESS charging power, respectively; $p_{i}^{\text {ed, min }}$ and $p_{i}^{\text {ed, } m a x}$ are the minimum and maximum limits of ESS discharging power, respectively; and $x_{t}^{\mathrm{ec}}$ and $x_{t}^{\text {ed }}$ are the binary variables representing ESS charging/discharging status, ESS is in the charging mode if $x_{t}^{\mathrm{ec}}=1$, and ESS is in the discharging mode if $x_{t}^{\text {ed }}=1$. Constraint (15) is the ESS energy balance constraint, where $E_{t}^{\text {es }}$ is the ESS SOC level, $\Delta t$ is the time interval of an hour, and $\eta^{c}$ and $\eta^{d}$ are the charging and discharging coefficients. Constraint (16) is the ESS SOC level constraint, where $E_{t}^{\mathrm{es}, \mathrm{min}}$ and $E_{t}^{\mathrm{es}, \mathrm{max}}$ are the minimum and maximum limits of the ESS SOC level.

\subsubsection{Bidirectional Converter Operation Constraint}

$$
\left\{\begin{array}{l}
0 \leq p_{t}^{\mathrm{atd}} \leq x_{t}^{\mathrm{atd}} p_{t}^{\mathrm{bc}, \max }, \quad \forall t \in N_{T}, \\
0 \leq p_{t}^{\mathrm{dta}} \leq x_{t}^{\mathrm{dta}} p_{t}^{\mathrm{bc}, \max }, \quad \forall t \in N_{T}, \\
x_{t}^{\mathrm{atd}}+x_{t}^{\mathrm{dta}}=1, \quad \forall t \in N_{T} .
\end{array}\right.
$$

Constraint (17) represents the transferring power limit, where $p_{t}^{\mathrm{bc}, \max }$ is the maximum active power converted on the $\mathrm{BC}$ and $x_{t}^{\text {atd }}$ and $x_{t}^{\mathrm{dta}}$ are binary variables representing the power conversion direction, power is transferred from the $\mathrm{AC}$ side to the DC side if $x_{t}^{\text {atd }}=1$, and power is transferred from the DC side to the AC side if $x_{t}^{\mathrm{dta}}=1$ and also represents that the BC can operate either in the charging mode or the discharging mode.

Finally, the energy management problem is formulated as a MIQP problem in (1)-(17). It is noted that the AC subproblem and DC subproblem are coupled through the DC conversion power $p_{t}^{\text {atd }}$ and $p_{t}^{\mathrm{dta}}$ in power balance equations (2) and (3) and constraint (17). In order to realize distributed energy management, the AC subgrid operator and DC subgrid operator should solve their own problems independently. Therefore, a P-ADMM-based algorithm is proposed to solve the energy management problem in a distributed manner, which will be detailed in the next section.

\section{P-ADMM-Based Solution Method}

In this section, the $\mathrm{P}-\mathrm{ADMM}$ algorithm is used to solve the optimal energy management problem in a distributed manner. Firstly, the MIQP problem is reformulated for the 
implementation of the P-ADMM algorithm. Then, the augmented Lagrangian of the problem is introduced. Finally, the P-ADMM-based algorithm is presented.
3.1. Decoupling of Coupled Constraints. To decouple power balance equations and $\mathrm{BC}$ operational constraints, a set of auxiliary variables are introduced as follows:

$$
\left\{\begin{array}{l}
p_{t}^{\mathrm{dta}}=\widehat{p}_{t}^{\mathrm{dta}}, p_{t}^{\mathrm{dta}}=\widetilde{p}_{t}^{\mathrm{atd}}, p_{t}^{\mathrm{atd}}=\widehat{p}_{t}^{\mathrm{atd}}, p_{t}^{\mathrm{atd}}=\widetilde{p}_{t}^{\mathrm{dta}}, \\
x_{t}^{\mathrm{atd}}=\widehat{x}_{t}^{\mathrm{atd}}, x_{t}^{\mathrm{dta}}=\widehat{x}_{t}^{\mathrm{dta}}, x_{t}^{\mathrm{dta}}=\widetilde{x}_{t}^{\mathrm{atd}}, x_{t}^{\mathrm{ec}}=\widehat{x}_{t}^{\mathrm{ec}}, x_{t}^{\mathrm{dc}}=\widehat{x}_{t}^{\mathrm{dc}} .
\end{array}\right.
$$

We replace original variables with auxiliary variables, and the model is reformulated.

Objective function of the reformulated model is (1).
Constraints of the reformulated model include original constraints (4)-(11), auxiliary constraint (18), and

$$
\begin{aligned}
& \widehat{x}_{t}^{\mathrm{ec}} p_{i}^{\mathrm{ec}, \min } \leq p_{t}^{\mathrm{ec}} \leq \hat{x}_{t}^{\mathrm{ec}} p_{i}^{\mathrm{ec}, \max }, \quad \forall t \in N_{T}, \\
& \widehat{x}_{t}^{\text {ed }} p_{i}^{\text {ed, min }} \leq p_{t}^{\text {ed }} \leq \widehat{x}_{t}^{\text {ed }} p_{i}^{\text {ed, } \max }, \quad \forall i \in N_{e}, t \in N_{T}, \\
& \hat{x}_{i, t}^{\mathrm{ec}}+\hat{x}_{i, t}^{\mathrm{ed}}=1, \quad t \in N_{T} \text {, } \\
& p_{t}^{\mathrm{UG}}+p_{t}^{\mathrm{DG}}+\eta_{\mathrm{dta}} \widetilde{\mathrm{p}}_{t}^{\mathrm{atd}}=p_{t}^{\mathrm{AC} \_L}+\widehat{p}_{t}^{\mathrm{atd}}, \quad \forall t \in N_{T}, \\
& p_{t}^{\mathrm{ed}}+p_{t}^{\mathrm{pv}}+\eta_{\mathrm{atd}} \tilde{p}_{t}^{\mathrm{dta}}=p_{t}^{e c}+\sum_{i \in N_{e}} p_{i, t}^{\mathrm{ev}}+\sum_{i \in N_{h}} p_{i, t}^{\mathrm{hp}}+\widehat{p}_{t}^{\mathrm{dta}}, \quad \forall t \in N_{T}, \\
& \begin{cases}0 \leq \hat{p}_{t}^{\text {atd }} \leq \widehat{x}_{t}^{\text {atd }} p_{t}^{\text {bc, max }}, & \forall t \in N_{T}, \\
0 \leq \widehat{p}_{t}^{\text {dta }} \leq \hat{x}_{t}^{\text {dta }} p_{t}^{\text {bc, max }}, & \forall t \in N_{T}\end{cases} \\
& \widehat{x}_{t}^{\mathrm{atd}}+\widetilde{x}_{t}^{\mathrm{atd}}=1, \quad \forall t \in N_{T} .
\end{aligned}
$$

It is assumed that variables with superscripts of "atd" are affiliated variables of the AC subgrid operator and variables with superscripts of "dta" are affiliated variables of the DC subgrid operator. Therefore, constraints (4)-(11) and (19)-(25) are fully decomposable between the AC and DC subgrids, whereas equality constraint (18) is still coupled.
3.2. Augmented Lagrangian. By adding equality constraint (18) into objective function (1) through dual variables $\Lambda=:\left\{\lambda_{t}, \widehat{\lambda}_{t}, \delta_{t}, \widehat{\delta}_{t}, \chi_{t}, \theta_{t}, \kappa_{t}, \psi_{t}\right\}$ and the penalty parameter $\rho$, the augmented Lagrangian of the MIQP model is formulated as follows:

$$
\begin{aligned}
& \min \sum_{t \in N_{T}} c_{1}^{G}\left(p_{t}^{\mathrm{DG}}\right)^{2}+c_{2}^{G} p_{t}^{\mathrm{DG}}+\lambda_{t} p_{t}^{\mathrm{UG}}+ \\
& \frac{\rho}{2}\left\{\left\|p_{t}^{\mathrm{dta}}-\widetilde{p}_{t}^{\mathrm{atd}}+\lambda_{t}\right\|^{2}-\left\|\lambda_{t}\right\|^{2}+\left\|p_{t}^{\mathrm{dta}}-\widehat{p}_{t}^{\mathrm{dta}}+\widehat{\lambda}_{t}\right\|^{2}-\left\|\widehat{\lambda}_{t}\right\|^{2}\right. \\
& \quad+\left\|p_{t}^{\mathrm{atd}}-\widetilde{p}_{t}^{\mathrm{dta}}+\delta_{t}\right\|^{2}-\left\|\delta_{t}\right\|^{2}+\left\|p_{t}^{\mathrm{atd}}-\widehat{p}_{t}^{\mathrm{atd}}+\widehat{\delta}_{t}\right\|^{2}-\left\|\widehat{\delta}_{t}\right\|^{2}+ \\
& \quad+\left\|x_{t}^{\mathrm{atd}}-\widehat{x}_{t}^{\mathrm{atd}}+\chi_{t}\right\|^{2}-\left\|\chi_{t}\right\|^{2}+\left\|x_{t}^{\mathrm{dta}}-\widehat{x}_{t}^{\mathrm{dta}}+\theta_{t}\right\|^{2}-\left\|\theta_{t}\right\|^{2}+ \\
& \left\|x_{t}^{\mathrm{dta}}-\widetilde{x}_{t}^{\mathrm{atd}}+\xi_{t}\right\|^{2}-\left\|\xi_{t}\right\|^{2}+\left\|x_{t}^{e c}-\widehat{x}_{t}^{e c}+\kappa_{t}\right\|^{2}-\left\|\kappa_{t}\right\|^{2}+ \\
& \left.\left\|x_{t}^{\text {ed }}-\widehat{x}_{t}^{\mathrm{ed}}+\psi_{t}\right\|^{2}-\left\|\psi_{t}\right\|^{2}\right\} .
\end{aligned}
$$


Subject to (4)-(11) and (19)-(25).

The augmented Lagrangian is optimized over two groups of primal variables $\mathbf{X}=:\left\{p_{t}^{\mathrm{DG}}, p_{t}^{\mathrm{UG}}, p_{i, t}^{\mathrm{ev}}, p_{i, t}^{\mathrm{hp}}, p_{t}^{\mathrm{ec}}, p_{t}^{\mathrm{ed}}, \widetilde{p}_{t}^{\mathrm{atd}}\right.$, $\left.\widehat{p}_{t}^{\mathrm{dta}}, \widetilde{p}_{t}^{\mathrm{dta}}, \widehat{p}_{t}^{\mathrm{atd}}, \hat{x}_{t}^{\mathrm{atd}}, \widetilde{x}_{t}^{\mathrm{atd}}, \widehat{x}_{t}^{\mathrm{dta}}, \widehat{x}_{t}^{\mathrm{ec}}, \widehat{x}_{t}^{\mathrm{ed}}\right\}$ and $\mathbf{Y}=:\left\{p_{t}^{\mathrm{dta}}, p_{t}^{\mathrm{atd}}\right.$, $\left.x_{t}^{\text {atd }}, x_{t}^{\text {dta }}, x_{t}^{\text {ec }}, x_{t}^{\text {ed }}\right\}$ and a group of dual variables $\Lambda$.

3.3. P-ADMM-Based Algorithm. For the ease of presentation, the objective function of the augmented Lagrangian is represented using a compact form $\mathbf{L}(\mathbf{X}, \mathbf{Y}, \Lambda)$. The augmented Lagrangian is solved by the P-ADMM-based algorithm with an iterative procedure, as shown in Algorithm 1, in which optimizations of two subproblems and the dualvariable update procedure are carried out iteratively.

3.4. Subproblem I. In the $k$ th iteration, primal variables $\mathbf{X}^{k}$ are optimized in subproblem I, given by (26), subject to (4)-(11) and (19)-(25), with parameters $\mathbf{Y}^{*, k-1}$ and $\Lambda^{* k-1}$ obtained in the $(k-1)$ th iteration.

$$
\text { Subproblem } \cdot \mathrm{I}: \cdot L\left(\mathbf{X}^{k}, \mathbf{Y}^{*, k-1}, \mathbf{\Lambda}^{*, k-1}\right) \text {, }
$$

s.t. constraints (4)-(11) and (19)-(25).

Subproblem I is a QP problem, and its objective function and constraints can be fully decoupled between AC and DC subgrids. Therefore, subproblem I can be solved in a distributed manner between AC and DC subgrids.

3.5. Subproblem II. The primal variables $\mathbf{Y}^{k}$ are optimized in subproblem II, given by (27) with parameters $\mathbf{X}^{*, k}$ and $\Lambda^{* k-1}$ obtained in the $k$ th and $(k-1)$ th iteration.

$$
\text { Subproblem } \cdot \text { II : } \cdot L\left(\mathbf{X}^{*, k}, \mathbf{Y}^{k}, \boldsymbol{\Lambda}^{*, k-1}\right) \text {. }
$$

It is noted that subproblem II is a nonconstrained MIQP problem. Instead of directly solving the MIQP problem to optimize binary variables, the binary variables are optimized using the projection function in (28), where $\mathbf{P}_{x}$ denotes the projection function, which rounds each entry to its nearest binary value. The remaining primal variables are optimized by solving the QP problem after excluding binary variables, which can be fully decoupled between AC and DC subgrids. Therefore, subproblem II can be solved in a distributed manner as well.

$$
\left\{\begin{array}{l}
x_{t}^{\mathrm{atd}}=\mathbf{P}_{x}\left(\hat{x}_{t}^{\mathrm{atd}}-x_{t}\right) \\
x_{t}^{\mathrm{dta}}=\mathbf{P}_{x}\left(\frac{\hat{x}_{t}^{\mathrm{dta}}-\theta_{t}+\tilde{x}_{t}^{\mathrm{atd}}-\xi_{t}}{2}\right)
\end{array}\right.
$$

3.6. Update of Dual Variables. After solving subproblems I and II, dual variables are updated in (29).

$$
\begin{aligned}
\Lambda^{k} & =\Lambda^{*, k-1}+\left(\mathbf{X}^{\mathrm{ex}, *, k-1}-\mathbf{Y}^{*, k}\right), \\
\mathbf{X}^{\mathrm{ex}} & =:\left\{\tilde{p}_{t}^{\text {atd }}, \widehat{p}_{t}^{\mathrm{dta}}, \widetilde{p}_{t}^{\mathrm{dta}}, \widehat{p}_{t}^{\text {atd }}, \widehat{x}_{t}^{\text {atd }}, \tilde{x}_{t}^{\text {atd }}, \widehat{x}_{t}^{\mathrm{dta}}\right\} .
\end{aligned}
$$

3.7. Stop Criteria. The iterative procedure continues till primal and dual residuals are lower than specified thresholds, respectively, as follows:

$$
\left\|\mathbf{X}^{\mathrm{ex}, *, k}-\mathbf{Y}^{*, k}\right\|^{2} \leq \sigma_{1},\left\|\mathbf{X}^{\mathrm{ex}, *, k}-\mathbf{X}^{\mathrm{ex}, *, k-1}\right\|^{2} \leq \sigma_{2} .
$$

\section{Case Studies}

A case study was conducted on the hybrid AC/DC system shown in Figure 1 to demonstrate the effectiveness of the proposed distributed energy management scheme. In the system, one DG and conventional loads are connected to the AC bus, whereas one ESS, 200 EVs [30, 32], $200 \mathrm{HPs}$, and one PV are connected to the DC bus. Figures 2 and 3 show the forecasted spot price and PV active power generation profile. The key parameters of DGs, EVs, HPs, and ESSs are given in Table 1 . The P-ADMM-based algorithm is implemented using GAMS [31, 33], and the solution is obtained with the CPLEX solver [34]. In the algorithm, convergence thresholds $\sigma_{1}$ and $\sigma_{2}$ are set as 0.01 , and penalty parameter $\rho$ is set as 50 .

4.1. Computational Performance. Although the convergence of the P-ADMM-based algorithm cannot be guaranteed for the MIQP model, the algorithm shows good convergence performance. A convergence analysis is conducted by solving the MIQP model with different penalty parameter values between 10 and 150 . Table 2 shows that the algorithm can converge within 650 iterations when the penalty parameter value varies within 10 and 150 . It can be seen that the convergence performance of the algorithm highly depends on the selection of the penalty parameter value. For example, an improper value $\rho=30$ would cause nonconvergence within the preset iteration limit (1000). Within the certain range, the algorithm with $\rho=50$ has the best convergence performance. The convergence process of the P-ADMM-based algorithm with $\rho=50$ is shown in Figure 4. It can be seen that the primal and dual residuals are lower than thresholds after 91 iterations.

The distributed solution $S^{\mathrm{d}}$ is compared with the centralized solution $\mathbf{S}^{\mathfrak{C}}$ in Table 3(take hour $t_{12}$ as an example). The centralized solution is obtained by solving the MIQP model using the GAMS/CPLEX solver with a zero optimality gap. It is shown in Table 2 that the distributed solution is almost the same as the centralized solution. The maximum relative error (RE) between the centralized and distributed solutions is $1.28 \%$ (the RE is calculated by (31)), which is very small and demonstrates that the proposed P-ADMM-based algorithm can reproduce the optimal centralized solution.

$$
\mathrm{RE}=\frac{\left\|\mathbf{S}^{\mathcal{c}}-\mathbf{S}^{d}\right\|}{\left\|\mathbf{S}^{c}\right\|} .
$$

4.2. Energy Management Results. Figures 5 and 6 show the optimal day-ahead energy schedules of the AC and DC subgrids, respectively. In the DC subgrid, the PV active 
P-ADMM algorithm

(1) input: system and DER parameters

(2) output: AC and DC energy schedules and BC operation status

(3) while: $\left\|\mathbf{X}^{\mathrm{ex}, *, k}-\mathbf{Y}^{*, k}\right\|^{2} \leq \sigma_{1},\left\|\mathbf{X}^{\mathrm{ex}, *, k}-\mathbf{X}^{\mathrm{ex}, *, k-1}\right\|^{2} \leq \sigma_{2}$ do

Solve subproblem I to update primal variables $\mathbf{X}^{k}$

Solve subproblem I to update primal variables $\mathbf{Y}^{k}$

Update dual variables $\Lambda^{k+1}$ using (30)

end

Algorithm 1: Pseudo-code of the P-ADMM algorithm.

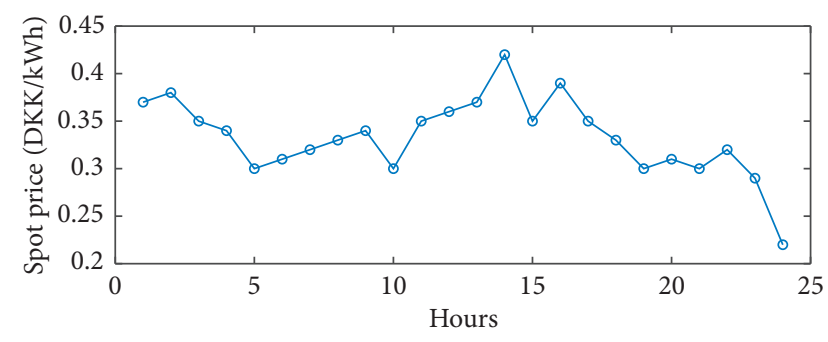

Figure 2: Forecasted spot prices.

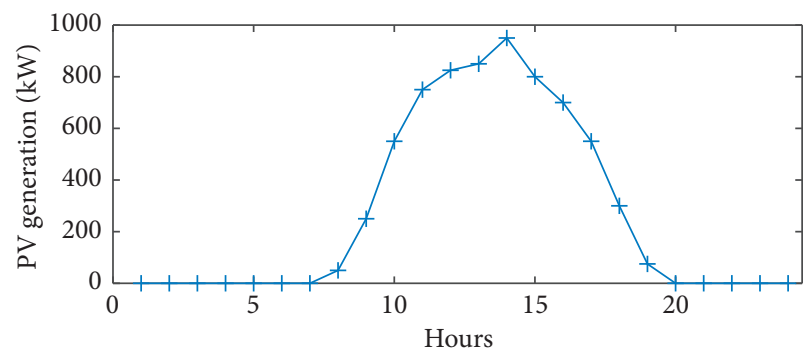

FIgURE 3: Forecasted PV active power generation.

power, ESS discharging power, and power converted from the AC subgird support the total power consumption of EVs and HPs. For load consumption, it can be seen that EVs and HPs tend to charge more power at hours with relatively low spot prices in order to minimize day-ahead energy costs. For power generations, in most hours, e.g., hours $t_{1}-t_{9}$ and $t_{19}-t_{24}, \mathrm{AC}$ power is converted into DC power to support EV and HP power consumption. At hours $t_{10}-t_{16}$, since there is sufficient PV active power, there is no need to convert AC power into DC power. Especially at hours $t_{14}$ and $t_{16}$, the DC subgrid delivers surplus power to the AC grid. In addition, the ESS would store surplus power at hours with relatively low prices and discharge power at those hours with relatively high spot prices to minimize energy costs.

In the AC subgrid, the $\mathrm{AC}$ operator chooses to dispatch the DG unit or buy external electricity for AC load consumption according to DG energy costs and spot prices. In this case, since the DG energy cost is smaller than the energy cost of purchasing external electricity in most hours, the DG maximum capacity is almost used. In addition, the external purchased electricity profile has almost opposite variation
TABLE 1: Key parameters used in simulations.

ESS parameters

ESS battery size $E_{t}^{\text {es,max }}$

Min/max charging power limit $p_{i}^{\text {ec,min }} / p_{i}^{\text {ec,max }}$

Min/max discharging power limit $p_{i}^{\text {ed,min }} / p_{i}^{\text {ed,max }}$

Charing/discharging coefficients $\eta^{c} / \eta^{d}$

kWh

$0 / 50 \mathrm{~kW}$

$0 / 50 \mathrm{~kW}$

$0.90 / 0.90$

\section{HP parameters}

COP of HP $c_{i}^{\text {cop }}$

$\mathrm{Min} / \max$ temperature of the house $K_{i, t}^{h, \min } / K_{i, t}^{h, \min }$

Min/max HP power consumption $p_{i, t}^{\mathrm{hp}, \min } / p_{i, t}^{\mathrm{hp}, \max }$

$20 / 24$ oC

\section{EV parameters}

Min/max HP power consumption $p_{i, t}^{e v, \min } / p_{i, t}^{\text {ev, } \max } \quad 0 / 11 \mathrm{~kW}$

EV availability $a_{i, t}$

\section{DG parameters}

Min/max DG active power generation

$p_{t}^{\mathrm{DG}, \mathrm{min}} / p_{t}^{\mathrm{DG}, \max }$

0/200 kW

Down- and upramping limits $p_{t}^{\mathrm{DG}, \mathrm{dr}} / p_{t}^{\mathrm{DG}, \mathrm{ur}}$

$80 \mathrm{~kW}$

DG energy costs $c_{1}^{G} / c_{2}^{G}$

$0.001 /$

0.15

\begin{tabular}{lc}
\hline \multicolumn{2}{c}{ BC parameters } \\
Max. limit of active power converted $p_{t}^{\text {bc,max }}$ & $1000 \mathrm{~kW}$ \\
Power transfer coefficients $\eta_{\mathrm{da}} / \eta_{\mathrm{dha}}$ & $0.9 / 0.9$
\end{tabular}

Power transfer coefficients $\eta_{\mathrm{dta}} / \eta_{\mathrm{dta}}$ $0.9 / 0.9$

TABle 2: Numbers of iterations with different penalty parameter values.

\begin{tabular}{lccccccc}
\hline$\rho$ & 10 & 20 & 30 & 40 & 50 & 100 & 150 \\
\hline Num. of iterations & 633 & 434 & - & 175 & 91 & 245 & 404 \\
\hline
\end{tabular}

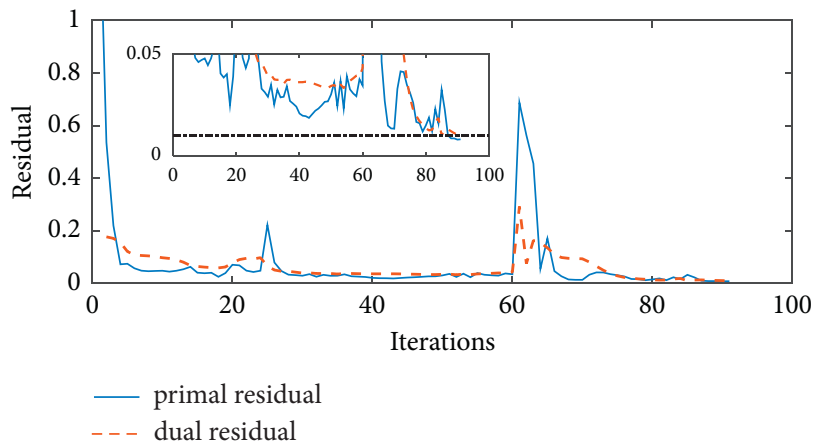

FIGURE 4: Convergence process of the P-ADMM-based algorithm $(\rho=50)$. 
TABle 3: Comparison between centralized and distributed solutions (take hour $t_{12}$ as an example).

\begin{tabular}{lccccccc}
\hline $\mathrm{kW}$ & $p_{t}^{\mathrm{DG}}$ & $\sum p_{i, t}^{\mathrm{ev}}$ & $\sum p_{i, t}^{\mathrm{hp}}$ & $p_{t}^{\mathrm{ec}}$ & $p_{t}^{\mathrm{ed}}$ & $p_{t}^{\mathrm{atd}}$ & $p_{t}^{\mathrm{dta}}$ \\
\hline Centralized & 139.9 & 219.2 & 559.5 & 46.2 & 0 & 0 & 0 \\
Distributed & 139.9 & 218.4 & 559.6 & 46.8 & 0 & 0 & 0 \\
\hline
\end{tabular}

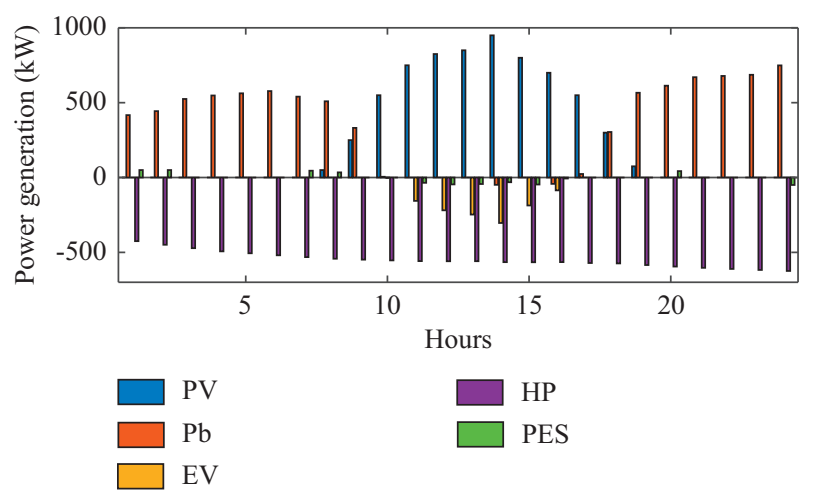

FIGURE 5: Day-ahead scheduling in the DC grid.

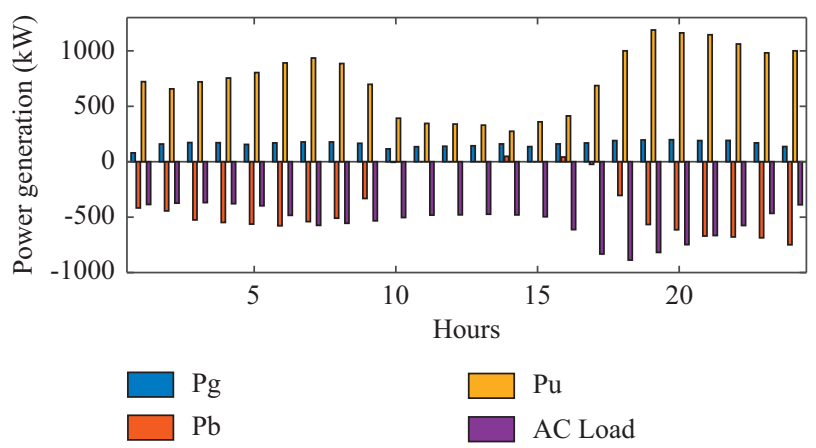

FIgUre 6: Day-ahead scheduling in the AC grid.

tendency as compared with the forecasted spot price profile in order to minimize energy costs.

\section{Conclusions}

To resolve the privacy information protection compromise and single-point failure issues when the centralized energy management scheme is applied, this paper proposes a distributed energy management scheme for the hybrid AC/DC microgrid based on the proposed P-ADMM-based algorithm. The energy management problem of the hybrid microgrid is formulated as a MIQP model, considering DER operation constraints, system operation constraints, and converter operation constraints. Then, the MIQP model is decomposed and distributed into smaller-scale submodels between subgrids using the P-ADMM-based algorithm. The numerical results demonstrate that the proposed algorithm can efficiently solve the MIQP model in a distributed manner and can deal with binary variables. The proposed distributed scheme allows each subgrid operator to make day-ahead schedules independently with information exchanges while acquiring the optimal energy management solution.

\section{Data Availability}

The data used to support the findings of this study are available from the corresponding author upon request.

\section{Conflicts of Interest}

The authors declare that there are no conflicts of interest regarding the publication of this paper.

\section{References}

[1] B. Kroposki, B. Johnson, Y. Zhang et al., "Achieving a 100\% renewable grid: operating electric power systems with extremely high levels of variable renewable energy," IEEE Power and Energy Magazine, vol. 15, no. 2, pp. 61-73, 2017.

[2] C. Wei, Z. Shen, D. Xiao, L. Wang, X. Bai, and H. Chen, "An optimal scheduling strategy for peer-to-peer trading in interconnected microgrids based on $\mathrm{RO}$ and nash bargaining," Applied Energy, vol. 275, 2021.

[3] C. Gamarra and J. M. Guerrero, "Computational optimization techniques applied to microgrids planning: a review," $R e$ newable and Sustainable Energy Reviews, vol. 48, pp. 413-424, 2015.

[4] H. M. A. Ahmed, A. B. Eltantawy, and M. M. A. Salama, "A planning approach for the network configuration of ac-dc hybrid distribution systems," IEEE Transaction on Smart Grid, vol. 9, no. 3, pp. 2203-2213, 2018.

[5] C. Zhao, S. Dong, C. Gu, F. Li, Y. Song, and N. P. Padhy, "New problem formulation for optimal demand side response in hybrid ac/dc systems," IEEE Transactions on Smart Grid, vol. 9, no. 4, pp. 3154-3165, 2018.

[6] Q. Jiang, M. Xue, and G. Geng, "Energy management of microgrid in grid-connected and stand-alone modes," IEEE Transactions on Power Systems, vol. 28, no. 3, pp. 3380-3389, 2013.

[7] A. Khodaei, "Microgrid optimal scheduling with multi-period islanding constraints," IEEE Transactions on Power Systems, vol. 29, no. 3, pp. 1383-1392, 2014.

[8] B. V. Solanki, C. A. Canizares, and K. Bhattacharya, "Practical energy management systems for isolated microgrids," IEEE Transactions on Smart Grid, vol. 10, no. 5, pp. 4762-4775, 2019.

[9] B. V. Solanki, A. Raghurajan, K. Bhattacharya, and C. A. Canizares, "Including smart loads for optimal demand response in integrated energy management systems for isolated microgrids," IEEE Transactions on Smart Grid, vol. 8, no. 4, pp. 1739-1748, 2017.

[10] X. Lu, N. Liu, Q. Chen, and J. Zhang, "Multi-objective optimal scheduling of a DC micro-grid consisted of PV system and EV charging station," in Proceedings of the 2014 IEEE Innovative Smart Grid Technologies - Asia (ISGT ASIA), pp. 487-491, Kuala Lumpur, Malaysia, May 2014.

[11] M. I. Ghiasi, M. A. Golkar, and A. Hajizadeh, "Lyapunov based-distributed fuzzy-sliding mode control for building integrated-DC microgrid with plug-in electric vehicle," IEEE Access, vol. 5, pp. 7746-7752, 2017.

[12] M. Dabbaghjamanesh, A. Kavousi-Fard, S. Mehraeen, J. Zhang, and Z. Y. Dong, "Sensitivity analysis of renewable energy integration on stochastic energy management of automated reconfigurable hybrid AC-DC microgrid considering 
DLR security constraint," IEEE Transactions on Industrial Informatics, vol. 16, no. 1, pp. 120-131, 2020.

[13] Z. Liang, H. Chen, X. Wang, S. Chen, and C. Zhang, "Riskbased uncertainty set optimization method for energy management of hybrid AC/DC microgrids with uncertain renewable generation," IEEE Transactions on Smart Grid, vol. 11, no. 2, pp. 1526-1542, 2020.

[14] M. Mahmoodi, P. Shamsi, and B. Fahimi, "Economic dispatch of a hybrid microgrid with distributed energy storage," IEEE Transactions on Smart Grid, vol. 6, no. 6, pp. 2607-2614, 2015.

[15] N. Eghtedarpour and E. Farjah, "Power control and management in a hybrid AC/DC microgrid," IEEE Transactions on Smart Grid, vol. 5, no. 3, pp. 1494-1505, 2014.

[16] B. Wei, X. Han, P. Wang, H. Yu, W. Li, and L. Guo, "Temporally coordinated energy management for AC/DC hybrid microgrid considering dynamic conversion efficiency of bidirectional AC/DC converter," IEEE Access, vol. 8, pp. 70878-70889, 2020.

[17] B. Zhao, H. Qiu, R. Qin, X. Zhang, W. Gu, and C. Wang, "Robust optimal dispatch of AC/DC hybrid microgrids considering generation and load uncertainties and energy storage loss," IEEE Transactions on Power Systems, vol. 33, no. 6, pp. 5945-5957, 2018.

[18] P. Teimourzadeh Baboli, M. Shahparasti, M. Parsa Moghaddam, M. R. Haghifam, and M. Mohamadian, "Energy management and operation modelling of hybrid AC-DC microgrid," IET Generation, Transmission \& Distribution, vol. 8, no. 10, pp. 1700-1711, 2014.

[19] J. Zhao, Q. Wu, N. Hatziargyriou, F. Li, and F. Teng, "Decentralized data-driven load restoration in coupled transmission and distribution system with wind power," IEEE Transactions on Power Systems, vol. 36, no. 5, pp. 4435-4444, 2021.

[20] J. Zhao, H. Wang, Q. Wu, N. D. Hatziargyriou, and F. Shen, "Optimal generator start-up sequence for bulk system restoration with active distribution networks," IEEE Transactions on Power Systems, vol. 36, no. 3, 2021.

[21] J. Zhao, H. Wang, Q. Wu, N. D. Hatziargyriou, and W. Zhang, "Spatio-temporal decomposition and coordination for distributed load restoration in AC/DC hybrid system," IEEE Transactions on Smart Grid, vol. 12, no. 2, 2021.

[22] F. Shen, Q. Wu, X. Jin, B. Zhou, C. Li, and Y. Xu, "ADMMbased market clearing and optimal flexibility bidding of distribution-level flexibility market for day-ahead congestion management of distribution networks," International Journal of Electrical Power \& Energy Systems, vol. 123, Article ID 106266, 2020.

[23] A. Silani and M. J. Yazdanpanah, "Distributed optimal microgrid energy management with considering stochastic load," IEEE Transactions on Sustainable Energy, vol. 10, no. 2, pp. 729-737, 2019.

[24] Q. Xu, T. Zhao, Y. Xu, Z. Xu, P. Wang, and F. Blaabjerg, “A distributed and robust energy management system for networked hybrid AC/DC microgrids," IEEE Transactions on Smart Grid, vol. 11, no. 4, pp. 3496-3508, 2020.

[25] F. Shen, Q. Wu, J. Zhao, W. Wei, N. D. Hatziargyriou, and F. Liu, "Distributed risk-limiting load restoration in unbalanced distribution systems with networked microgrids," IEEE Transactions on Smart Grid, vol. 11, no. 6, pp. 4574-4586, 2020.

[26] F. Shen, J. C. López, Q. Wu, M. J. Rider, T. Lu, and N. D. Hatziargyriou, "Distributed self-healing scheme for unbalanced electrical distribution systems based on alternating direction method of multipliers," IEEE Transactions on Power Systems, vol. 35, no. 3, pp. 2190-2199, 2020.

[27] J. C. López, M. J. Rider, F. Shen, and Q. Wu, "Distributed service restoration of active electrical distribution systems using ADMM," in Proceedings of the 2019 IEEE Power \& Energy Society Innovative Smart Grid Technologies Conference (ISGT), pp. 1-5, Washington, DC, USA, February 2019.

[28] F. Shen, Q. Wu, Y. Xu, F. Li, F. Teng, and G. Strbac, "Hierarchical service restoration scheme for active distribution networks based on ADMM," International Journal of Electrical Power \& Energy Systems, vol. 118, Article ID 105809, 2020.

[29] Q. Zhang, K. Dehghanpour, and Z. Wang, "Distributed CVR in unbalanced distribution systems with PV penetration," IEEE Transactions on Smart Grid, vol. 10, no. 5, pp. 53085319, 2019.

[30] S. Huang, Q. Wu, S. S. Oren, R. Li, and Z. Liu, “Distribution locational marginal pricing through quadratic programming for congestion management in distribution networks," IEEE Transactions on Power Systems, vol. 30, no. 4, pp. 2170-2178, 2015.

[31] C. Wei, M. Benosman, and T. Kim, "Online parameter identification for state of power prediction of lithium-ion batteries in electric vehicles using extremum seeking," International Journal of Control, Automation and Systems, vol. 17, no. 11, pp. 2906-2916, 2019.

[32] P. Bacher and H. Madsen, "Identifying suitable models for the heat dynamics of buildings," Energy and Buildings, vol. 43, no. 7, pp. 1511-1522, 2011.

[33] GAMS. Language and Environment, https://www.gams.com/ latest/docs/UG_MAIN.html\#UG_Language_Environment.

[34] Overview of CPELX, https://www.gams.com/latest/docs/S_ CPLEX.html. 